\title{
Spectrophotometric Simultaneous Determination of Caffeine and Paracetamol in Commercial Pharmaceutical by Principal Component Regression, Partial Least Squares and Artificial Neural Networks Chemometric Methods
}

\author{
A. Hakan Aktaş* and Filiz Kitiş \\ Süleyman Demirel University, Science and Art Faculty, Department of Chemistry, 32260 Isparta - Turkey \\ RECEIVED NOVEMBER 20, 2012; REVISED NOVEMBER 19, 2013; ACCEPTED JANUARY 8, 2014

\begin{abstract}
Three multivariate calibration-prediction techniques, principal component regression (PCR), partial least squares (PLS) and artificial neural networks (ANN) were applied to the spectrometric multicomponent analysis of the drug containing paracetamol (PCT) and caffeine (CAF) without any separation step. The selection of variables was studied. A series of synthetic solution containing different concentrations of PCT and CAF were used to check the prediction ability of the PCR, PLS and ANN. The results obtained in this investigation strongly encourage us to apply these techniques for a routine analysis and quality control of the drug.(doi: 10.5562/cca2214)
\end{abstract}

Keywords: paracetamol, caffeine, spectrometry, multivariate calibration

\section{INTRODUCTION}

Paracetamol (PCT) and caffeine (CAF) are active substance widely employed in pharmaceutical preparation and are frequently combined in the same medicine. PCT is a popular antipyretic and analgesic agent. ${ }^{1} \mathrm{CAF}$, a methylated xanthine and potent stimulant of the central nervous system, has been added to PCT and CAF in various combinations. Worked in the chemical structures of these two substances are shown in Figure 1.

Therefore, the interest in the development of simultaneous analysis methods, which do not evolve a preliminary separation step, suited for routine pharmaceutical analysis is justified. Various methods, including official methods, ${ }^{2-4}$ spectrophotometry, ${ }^{5-8}$ and chromatography ${ }^{9,10}$<smiles>Cn1c(=O)c2c(ncn2C)n(C)c1=O</smiles>

Caffeine<smiles>CC(=O)Nc1ccc(O)cc1</smiles>

Paracetamol
Figure 1. Structures of the two studied compounds. are available for the determination of above compounds, whether alone or in combination with other drugs.

The quality control of dosage form preparations of drug requires reliable and quick analytical methods. UV/VIS spectrophotometry is by far the instrumental technique of choice of industrial laboratories, owing mainly to simplicity, often demanding low cost equipment. Simultaneous quantitative analysis of pharmaceuticals containing multi-active compounds is difficult to perform by classical spectrophotometric method due to overlapping spectra. ${ }^{11}$ In recent years, multivariate chemometric methods seem to be the techniques showing a best performance in terms of complex mixture solution. ${ }^{12-15}$ The same methods and their algorithms have been applied to the simultaneous spectrophotometric determination of drugs in the pharmaceutical formulations containing two or more compounds with overlapping spectra. The most adopted multivariate methods in pharmaceutical analysis are principal component regression (PCR) and partial least-squares regression (PLS), whose theory has allowed to establish a relation between matrices of chemical data. ${ }^{16-18}$ This model is used in a second step to predict the composition of a sample containing the same components with unknown concentrations. Artificial neural networks (ANN) are powerful chemometric methods as they do not need any model structure specification and can process multivariate prob-

\footnotetext{
* Author to whom correspondence should be addressed. (E-mail: hakanaktas@sdu.edu.tr)
} 
lems of non-linear systems. Therefore, ANN has attracted many researchers' attentions. ${ }^{19-22}$ The corresponding nonlinear multivariate maps use a non-linear transformation of the input variable to project inputs onto designated attribute values in output space. The strength of modeling with layered, feed-forward ANNs lies in the flexibility of the distributed soft model defined by the weight of the network. Both linear and non-linear mapping functions can be modeled by suitably configuring the network. The multilayer feed-forward neural network trained with back-propagation learning algorithm becomes an increasingly popular technique. ${ }^{23-25}$ Recently, we reported the application of ANN for non-linear calibration by using potentiometric titration and spectrophotometry. ${ }^{26-28}$

In this study, three chemometric methods were successfully applied to simultaneous determination of PCT and CAF in a commercial tablet formulation, tablets without any separation procedure. The chemometric calibrations were carried out by using the mixtures of these pharmaceutical tablets in suitable and possible compositions and concentrations. Means recoveries (\%) and relative standard deviation of PCR, PLS and ANN methods were calculated for the validation of the methods. Amounts of the dissolved tablets were calculated by these chemometric methods and dissolution profiles were revealed. The obtained results were statistically compared each other.

\section{EXPERIMENTAL}

\section{Apparatus and Software}

An absorbance measurement was carried out by using a Shimadzu (Model UV-1700) UV-Visible spectrometer (Shimadzu, Kyoto, Japan), equipped with $1 \mathrm{~cm}$ matched quartz cells, and was used for spectrometric measurements. Application of PCR and PLS algorithms was supported by the software package "Minitab ${ }^{\circledR} 16$ ". The software is dedicated to both multivariate analysis and experimental design and is equipped with several multivariate methods. It allows to optimize the calibration models and to develop validation procedures.

The back-propagation neural network algorithm three layers were used in MATLAB (version 7.0, Math Work Inc.) using NN toolbox. All programs were run on a Pentium, personal computer, with windows XP home edition.

\section{Commercial Product}

A commercial pharmaceutical product (Vermidon ${ }^{\circledR}$ Oral tablet, Sandoz Pharm. Ind., Kocaeli, Turkey) was purchased from local resources and assayed. Its declared content was as follows: Paracetamol $500 \mathrm{mg}$ and caffeine $30 \mathrm{mg}$, in each capsule.

\section{Chemicals}

Paracetamol and caffeine were kindly donated by the

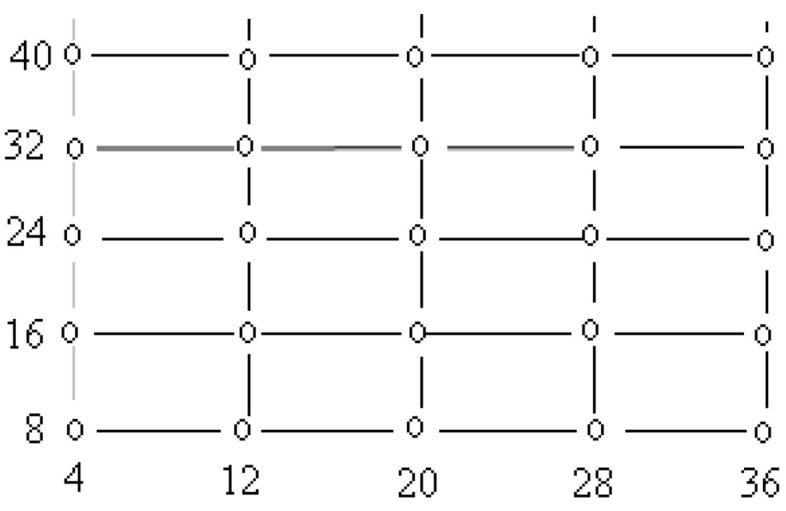

Figure 2. Concentration set design for the preparation of PCR, PLS and ANN calibrations.

pharmaceutical industries and were used without further purification. All solvents and reagents were of analytical reagent grade (Sigma and Fluka).

\section{Standard Solutions}

Stock solution $50 \mathrm{mg} / 100 \mathrm{~mL}$ PCT and CAF in $0.1 \mathrm{M}$ $\mathrm{HCl}$ were used to set up the calibration set samples. A concentration set of 25 mixture solutions consisting of $\mathrm{PCT}$ and CAF in the concentration range of 8.0-40.0 and $4.0-36.0 \mu \mathrm{g} / \mathrm{mL}$ for PCT and CAF in the same solvent were symmetrically prepared from the prepared stock solutions respectively (Figure 2). Symmetric set of calibration is preferred. The reason for this is to minimize errors in calibration may occur during analysis. To check the proposed methods we used an independent validation set consisting of the synthetic mixture solutions of PCT and CAF in the above working concentration ranges.

\section{Sample Solutions}

Pharmaceutical formulations were assayed by weighing the content of 20 tablets and reducing them a fine powder. An amount exactly corresponding to the average tablet weight was suspended in $0.1 \mathrm{M} \mathrm{HCl}$ and made up to a volume of $100 \mathrm{~mL}$ calibrated flasks. The suspension of the flask was mechanically shaken for $30 \mathrm{~min}$ and filtrated into a $100 \mathrm{~mL}$ volumetric flask through a 0.45 $\mu \mathrm{m}$ membrane filter. The final tablet solution was diluted to the working concentration range. The absorption spectra of these sample solution were recorded for the application of the PCR, PLS and ANN calibrations.

\section{Chemometric Methods}

In this paper only outlines of the three chemometric methods will be provided, detailed mathematical treatments as well as applications tutorials may be found in the referenced literature.

$P C R$ and PLS

$\mathrm{PCR}^{29-31}$ and $\mathrm{PLS}^{32,33}$ are factor analysis multivariate statistical tools which have many of the full spectrum 
Table 1. Calibration set from symmetrically mixture design

\begin{tabular}{lcrrrr}
\hline & Concentration $(\mu \mathrm{g} / \mathrm{mL})$ & \multicolumn{3}{c}{ Concentration $(\mu \mathrm{g} / \mathrm{mL})$} \\
\hline No. & CAF & PCT & No. & CAF & PCT \\
\hline 1 & 4.0 & 8.0 & 14 & 20.0 & 32.0 \\
2 & 4.0 & 16.0 & 15 & 20.0 & 40.0 \\
3 & 4.0 & 32.0 & 16 & 28.0 & 8.0 \\
4 & 4.0 & 40.0 & 17 & 28.0 & 16.0 \\
5 & 4.0 & 8.0 & 18 & 28.0 & 24.0 \\
6 & 12.0 & 16.0 & 19 & 28.0 & 32.0 \\
7 & 12.0 & 24.0 & 20 & 28.0 & 40.0 \\
8 & 12.0 & 32.0 & 21 & 36.0 & 8.0 \\
9 & 12.0 & 40.0 & 23 & 36.0 & 16.0 \\
10 & 12.0 & 16.0 & 24 & 36.0 & 24.0 \\
11 & 20.0 & 24.0 & 25 & 36.0 & 32.0 \\
12 & 20.0 & & & 36.0 & 40.0 \\
13 & 20.0 & & & & \\
\hline
\end{tabular}

advantages and have been successfully applied to spectrophotometric analyses of multicomponent mixtures. PCR and PLS need a calibration step where the models for the spectra and the component concentrations of the unknown are estimated from the sample spectrum. Both of these methods involve spectral decomposition.

The PCR decomposition is based entirely on spectral variations without regard for the component concentrations. In PLS, the spectral decomposition is weighted to the concentration. The major difference in the predictive abilities of these two methods is that PLS seems to predict better than PCR when there are random linear baselines or independently varying major spectral components which overlap with the spectral features of the analysis. The optimal of calibration method depend on the particular experimental conditions. However, PLS seems to a reasonable choice over a wide range of conditions.

$A N N$

The neural network represents a powerful data modeling tool that is capable to capture and describe complex input/output relationships. The true power and advantage of neural networks consist in their ability to represent both linear and non-linear relationships and in their ability to learn these relationships directly by using the data being modeled. The optimal topological neural network is performed for the calibration set to provide high recovery results with small relative error.

\section{RESULTS AND DISCUSSION}

\section{Method Development}

Figure 3 displays the UV absorption spectra of PCT and CAF together with their mixture. In the presence of the overlapping spectra of two compounds in the spectral range $205-305 \mathrm{~nm}$. Spectrum as shown the simultaneous determination of the related compounds in samples is not possible by using classical spectrophotometric approaches. We have focused mainly on the quantitative resolution of the binary mixtures of PCT and CAF by using PCR, PLS and ANN chemometric approaches without any separation step.

PCR, PLS and ANN Methods

The first step in simultaneous determination of the binary mixture of drugs by multivariate calibration methods involves constructing the calibration matrix for binary mixture of PCT and CAF. To build PCR, PLS and ANN calibration models, a concentration set of 25 mixtures of two compounds in the range of $8.0-40.0 \mu \mathrm{g} / \mathrm{mL}$ for PCT and $4.0-36.0 \mu \mathrm{g} / \mathrm{mL}$ for CAF in $0.1 \mathrm{M} \mathrm{HCl}$ was symmetrically prepared as seen in Figure 2 . The concentration set and its composition corresponding to Figure 3 were presented in Table 1.

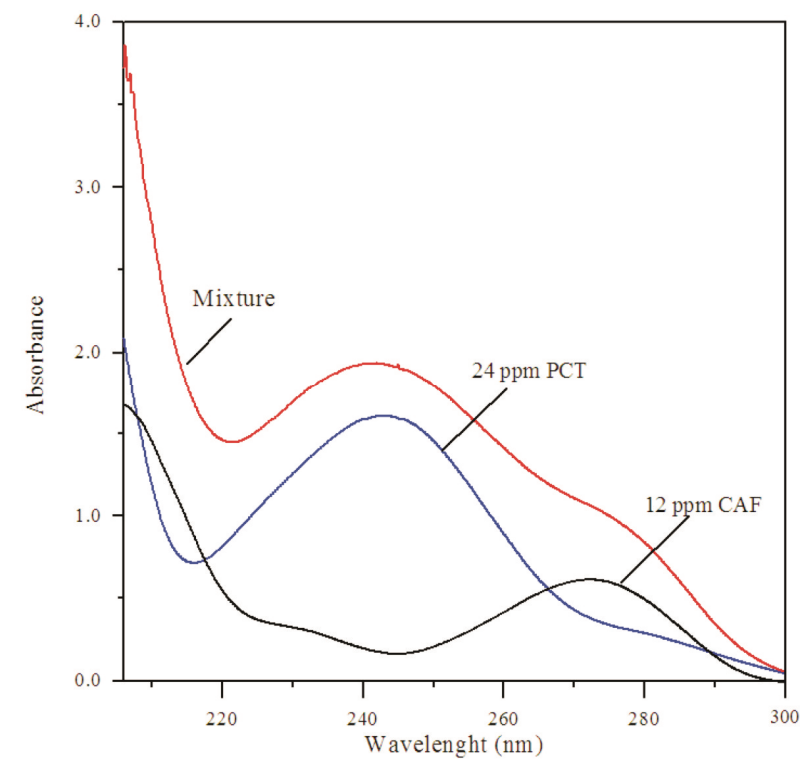

Figure 3. Absorption spectra of 24 ppm PCT, 12 ppm CAF and their mixture in $0.1 \mathrm{M} \mathrm{HCl}$. 
Table 2. Statistical parameters for PCR, PLS and ANN

\begin{tabular}{|c|c|c|c|c|c|c|c|}
\hline \multirow[b]{2}{*}{ Step } & \multirow[b]{2}{*}{ Parameter } & \multicolumn{2}{|c|}{ PCR } & \multicolumn{2}{|c|}{ PLS } & \multicolumn{2}{|c|}{ ANN } \\
\hline & & PCT & CAF & PCT & CAF & PCT & CAF \\
\hline \multirow[t]{5}{*}{ Calibration } & SEC & 0.1583 & 0.0875 & 0.1154 & 0.0408 & - & - \\
\hline & PRESS & 0.5029 & 0.7423 & 0.7304 & 0.4428 & 0.9180 & 1.7201 \\
\hline & SLOPE & 0.9965 & 1.0021 & 0.9994 & 0.9996 & 1.0099 & 1.0162 \\
\hline & Intercept & 0.1460 & 0.0422 & 0.0177 & 0.0075 & 0.0188 & 0.2625 \\
\hline & $r$ & 0.9996 & 0.9997 & 0.9997 & 0.9994 & 0.9998 & 0.9991 \\
\hline \multirow[t]{4}{*}{ Prediction } & SEP & 0.2224 & 0.1831 & 0.2206 & 0.1718 & 0.2473 & 0.3386 \\
\hline & Slope & 0.9982 & 0.9994 & 0.9981 & 0.9998 & 1.0102 & 1.0015 \\
\hline & Intercept & 0.0235 & 0.0059 & 0.055 & 0.0424 & 0.1408 & 0.0862 \\
\hline & $r$ & 0.9998 & 0.9997 & 0.9998 & 0.9998 & 0.9988 & 0.9996 \\
\hline
\end{tabular}

The absorption values of spectra of the concentration set were measured at the wavelength set with $\Delta \lambda=$ $0.1 \mathrm{~nm}$ in the spectral region of 205-305 $\mathrm{nm}$. The concentration set and absorption data were considered as $y$ block $(25 \times 2)$ x-block $(25 \times 1000)$ for the construction PCR and PLS chemometric calibration. During the calibration process various factors were tested by using cross validation procedure to obtain the best recovery results. The calculations were done within PLS Toolbox 4.1 and $w$ have selected the optimal factor to be 5 .

In the application of the ANN approach, the investigation of the optimal topological neural network for the calibration set was performed. The first step was to prepare the input for the topological neural network. This input consist of 25 spectra consisting 20 points and different 25 concentrations corresponding to the calibration set as can be seen in Table 1 . The main objective was to find the appropriate topological neural structure which allow us to obtain a high recovery result. We found out a logsig input layer together with two hidden layers consisting 5 and 4 neurons, respectively and two output layers, was chosen as the optimal configuration. A logsig transfer function was used for each layer.

The application competence of a calibration model can be explained in several ways. We can also examine these results numerically. One of the best ways to do this, by examining the predicted residual error sum of squares (PRESS). To calculate PRESS we compute the errors between the expected and predicted values for all the samples, square them, and sum them together.

$$
\operatorname{PRESS}=\sum_{i=1}^{n}\left(C_{i}^{\text {added }}-C_{i}^{\text {found }}\right)^{2}
$$

Strikingly speaking, this is not a correct way to normalize the PRESS values when not all of the data sets contain the same number of samples. If we want correctly compare PRESS values for data sets that contain differing numbers of samples, we should convert to standard error of prediction (SEP), which is given by following formula.

$$
\mathrm{SEP}=\sqrt{\frac{\sum_{i=1}^{n}\left(C_{i}^{\text {added }}-C_{i}^{\text {found }}\right)^{2}}{n}}
$$

Where $C_{i}^{\text {added }}$ the added concentration of drug is, $C_{i}^{\text {found }}$ is the found concentration of drug and $n$ is the total number of the synthetic mixtures. The SEP can provide a good measure of how well, on average, the calibration model performs. Often, however, the performance of the calibration model varies depending on the analyte level.

The standard error of calibration denoted by SEC represents another important quantity and is given us by

$$
\mathrm{SEC}=\sqrt{\frac{\sum_{i=1}^{n}\left(C_{i}^{\text {added }}-C_{i}^{\text {found }}\right)^{2}}{n}}
$$

The values of PRESS, SEP and SEC were calculated and the results are presented in Table 2 . In the same table, the statistical parameters between actual and predicted concentrations of CFT and CAF substances in mixtures were found by using the experimental data in the calibration and prediction steps.

\section{Method Validation}

The validation of PCR, PLS and ANN methods have been done by their performance for obtaining reliable results of analysis. Therefore, 15 synthetic mixtures containing PCT and CAF in different concentrations levels as shown in Table 3 were prepared as an independent validation set. The percentage recoveries and relative standard deviations were indicated in Table 3. In the recovery study, the numerical values were found satisfactory for the validity PCR, PLS and ANN. The reliable accuracy and higher precision in application of 
Table 3. Recovery results obtained in synthetic mixtures for PCR, PLS and ANN methods

\begin{tabular}{|c|c|c|c|c|c|c|c|}
\hline \multirow{2}{*}{\multicolumn{2}{|c|}{ Mixtures added $(\mu \mathrm{g} / \mathrm{mL})$}} & \multicolumn{6}{|c|}{ Recovery / \% } \\
\hline & & \multicolumn{2}{|c|}{ PCR } & \multicolumn{2}{|c|}{ PLS } & \multicolumn{2}{|c|}{ ANN } \\
\hline PCT & $\mathrm{CAF}$ & PCT & $\mathrm{CAF}$ & PCT & $\mathrm{CAF}$ & PCT & CAF \\
\hline 4 & 8 & 100.63 & 99.25 & 96.75 & 100.75 & 101.25 & 99.24 \\
\hline 12 & 16 & 101.75 & 101.08 & 102.31 & 99.75 & 102.32 & 100.12 \\
\hline 20 & 24 & 100.96 & 99.85 & 100.63 & 99.80 & 100.32 & 101.50 \\
\hline 28 & 32 & 100.25 & 100.14 & 100.03 & 100.11 & 100.35 & 100.25 \\
\hline 36 & 40 & 104.38 & 100.03 & 100.03 & 99.92 & 101.13 & 102.10 \\
\hline 20 & 8 & 96.94 & 101.20 & 104.25 & 101.35 & 102.55 & 100.12 \\
\hline 20 & 16 & 99.17 & 101.95 & 97.19 & 98.55 & 99.80 & 101.18 \\
\hline 20 & 24 & 99.95 & 99.80 & 99.33 & 100.00 & 100.00 & 100.21 \\
\hline 20 & 32 & 99.84 & 101.65 & 99.85 & 100.95 & 100.30 & 99.88 \\
\hline 20 & 40 & 99.95 & 100.65 & 100.00 & 100.25 & 101.35 & 101.20 \\
\hline 4 & 24 & 101.29 & 96.75 & 99.84 & 96.25 & 100.50 & 101.00 \\
\hline 12 & 24 & 100.38 & 99.00 & 101.21 & 99.75 & 101.00 & 100.25 \\
\hline 20 & 24 & 99.83 & 101.60 & 99.00 & 101.85 & 100.60 & 100.33 \\
\hline 28 & 24 & 101.25 & 100.32 & 100.46 & 99.96 & 100.86 & 101.08 \\
\hline 36 & 24 & 100.08 & 99.83 & 99.83 & 99.56 & 101.33 & 97.17 \\
\hline Mean & & 100.45 & 100.21 & 100.05 & 99.85 & 100.91 & 100.37 \\
\hline $\operatorname{RSD}^{(a)}$ & & 1.56 & 1.26 & 1.56 & 1.27 & 0.78 & 1.15 \\
\hline
\end{tabular}

Table 4. Recovery results obtained standard addition technique by the proposed PCR, PLS and ANN methods

\begin{tabular}{ccccccccc}
\hline \multicolumn{2}{c}{} & \multicolumn{2}{c}{ PCR } & \multicolumn{2}{c}{ Recovery $/ \%$} & \multicolumn{3}{c}{ ANN } \\
Added to tablet $(\mu \mathrm{g} / \mathrm{mL})$ & $\mathrm{CAF}$ & PCT & CAF & PCT & CAF & PCT & CAF \\
PCT & CAF & 98.33 & 99.67 & 96.00 & 95.67 & 98.67 & 99.33 \\
3.0 & 6.0 & 99.00 & 99.00 & 96.33 & 97.17 & 98.00 & 98.67 \\
6.0 & 12.0 & 96.88 & 98.22 & 98.67 & 96.87 & 99.11 & 99.22 \\
9.0 & 18.0 & 97.07 & 98.96 & 97.00 & 96.87 & 98.59 & 99.07 \\
& Mean & RSD & 1.84 & 0.72 & 1.45 & 1.08 & 0.55 & 0.35 \\
\hline
\end{tabular}

both compounds. During the process of the analysis, interference and systematical errors were absent.

To test the selectivity of the methods, the standard of PCT and CAF was added to the tablet solution. This procedure was repeated five times for each concentration level. During the process no interference of the excipients formulation was reported. Therefore, PCR, PLS and ANN methods apply to proposed in this study are appropriate for the determination of PCT and CAF compounds in the tablets. The recovery results presented in Table 4.

Table 5. Assay results for the commercial pharmaceutical formulation (mg/tablet)

\begin{tabular}{ccccccc}
\hline & \multicolumn{2}{c}{ PCR } & \multicolumn{2}{c}{ PLS } & \multicolumn{2}{c}{ ANN } \\
\hline No & PCT & CAF & PCT & CAF & PCT & CAF \\
\hline 1 & 505.8 & 29.9 & 500.0 & 29.8 & 500.0 & 30.5 \\
2 & 495.2 & 30.6 & 499.0 & 29.1 & 498.9 & 30.3 \\
3 & 503.0 & 28.5 & 494.8 & 27.8 & 499.2 & 29.8 \\
4 & 519.2 & 27.5 & 505.3 & 29.4 & 502.8 & 26.8 \\
5 & 496.8 & 29.8 & 502.8 & 32.8 & 501.4 & 29.8 \\
6 & 499.2 & 29.6 & 498.1 & 28.9 & 494.8 & 32.0 \\
7 & 502.8 & 30.2 & 499.2 & 29.5 & 499.8 & 29.5 \\
8 & 501.3 & 29.1 & 500.2 & 30.8 & 508.1 & 31.0 \\
9 & 492.7 & 31.3 & 498.8 & 32.2 & 500.3 & 30.0 \\
10 & 498.7 & 28.9 & 499.9 & 30.4 & 499.1 & 29.8 \\
Mean & 499.9 & 29.55 & 499.5 & 29.95 & 500.25 & 30.15 \\
RSD & 6.19 & 1.3 & 1.39 & 1.7 & 2.29 & 0.69 \\
\hline
\end{tabular}


Analysis of Commercial Pharmaceutical

Determination results obtained by the application of PCR, PLS and ANN calibrations to the tablet solutions containing PCT and CAF in Vermidon ${ }^{\circledR}$ Oral tablet formulations were summarized in Table 5. The analysis obtained from three methods was found satisfactory for the quantitative analysis of commercial tablet. Moreover, proposed procedures gave results in agreement with the labeled drugs content when applied on pharmaceutics.

\section{CONCLUSIONS}

Three chemometric technique in spectrometric analysis, PCR, PLS and ANN were proposed for the simultaneous determination of PCT and CAF in their binary mixtures. These techniques were applied with great success to commercial pharmaceutical tablets. The resolution of highly overlapping drug mixtures was achieved by the use of PCR, PLS and ANN techniques. A selection of working wavelength having high correlation values with concentration due to interference coming from matrix sample or additional analytes outside the working range. The proposed chemometric techniques can be applied for the routine analysis of drug in the tablet formulation without any a priori chemical separation and without time consuming.

Acknowledgements. We would like to thank to research council of Süleyman Demirel University of Turkey for financial support of this investigation.

\section{REFERENCES}

1. J. G. Hardman, L. E. Limbard, P. B. Molinoff, R. W. Ruddon, A. Goodman - Gilman, Gilman's The Pharmacological Basis of Therapeutics, Ninth ed., McGraw-Hill New York, 1996.

2. U.S. Pharmacopoeia, XX, American Pharmaceutical Association, Washington DC, 1975.

3. European Pharmacopoeia, fourth ed., Council of Europe, Strasbourg, 2002.

4. British Pharmacopoeia, Her Majesty's Stationary Office, London, 2005.
5. R. D. Bautista, A. I. Jimenez, and J. J. Arias, Anal. Lett. 29 (1996) 2645.

6. M. J. A. Canada, M. I. P. Reguera, A. M. Diaz, and L. F. Capitan-Valley, Talanta 49 (1999) 691.

7. A. D. Vidal, J. F. G. Reyes, P. O. Barrales, and A. M. Diaz, Anal. Lett. 35 (2002) 2433.

8. E. Dinç, A. Özdemir, and D. Baleanu, Talanta 65 (2005) 36.

9. L.Suntornsuk, O. Pipitharome, and P. Wilairat, J. Pharm. Biomed. Anal. 33(2003) 441.

10. G. B. Golubitskii, E. V. Budka E. M. Basova, V. M. Ivanov, and A. V. Kostarnoi, J. Anal. Chem. 62 (2007) 571.

11. M. R. Khoshayand, H. Abdollahi, M. Shariatpanahi, A. Saadatfard, and A. Mohammadi, Spectrochi. Acta Part A $\mathbf{7 0}$ (2008) 491.

12. B. Lavine, Anal. Chem. 72 (2000) 91.

13. R. Brereton, Chemometrics Data Analysis for the Laboratory and Chemical Plant, Wiley, Chichester, 2003.

14. G. Ragno, G. Ioele, and A. Risali, Anal. Chim. Acta 512 (2004) 173.

15. A. H. Aktaş and S. Göksu, Reviews in Analytical Chemistry 29(2) (2010) 81 .

16. K. R. Beebe and B. R. Kowalski, Anal. Chem. 59 (1987) 1007A

17. H. Martens and T. Naes, Multivariate Calibration, Wiley, Chichester, 1988.

18. H. Wold, H. Martens, and S. Wold, in: A. Ruhe and B. Kagstrom (Eds.), Multivariate Calibration Problems in Chemistry Solved by PLS, Heidelberg, (1983) 286.

19. L. Zupan and J.Gastreiger, Anal. Chim. Acta 248 (1991) 1.

20. T. B. Blank and S. D. Brown, Anal. Chem. 65 (1993) 3081.

21. F. Despagne and D. L. Massart, Analyst 123 (1998) 157R.

22. C. Ruckebusch, L. Duponchel, and J. L. Huvenne, Chemom. Intell. Lab. Syst. 62 (2002) 189.

23. S. Sehulic, M. B. Seasholtz, Z. Wang, B. R. Kowalski, S. E. Lee, and B. R. Holt, Anal. Chem. 65 (1993) 835A.

24. W. Wu, B. Walczak, D. L. Massart, S. Heuerding, F. Erni, I. R. Lost, and K. A. Prebble, Anal. Chim. Acta 33 (1996) 35.

25. C. Broggaard and H. H. Thodberg, Anal. Chem. 64 (1992) 545.

26. A. H. Aktaş and S. Yaşar, Acta Chim. Slov. 51(2) (2004) 273.

27. A. H. Aktaş and G. P. Ertokuş, J. of Serbian Chem. Soc. 73(1) (2008) 87.

28. A. H. Aktaş and S. Aydoğdu, Chemia Analityzna 54(4) (2009) 795.

29. I. T. Jolliffe, Principal Component Analysis, Springer, New York, 1986.

30. P. M. Fredericks, J. B. Lee, P. R. Osborn, and D. A. J. Swenkeis, Appl. Spectrosc. 39 (1985) 303.

31. C. J. Brown, R. J. Obremski, and P. Anderson, Appl. Spectrosc. 40 (1986) 734

32. M. Otto and W. Wegscheider, Anal. Chem. 57 (1985) 63.

33. P. Geladi and B. R. Kowalski, Anal. Chim. Acta 185 (1986) 1. 